\title{
The effects of acupressure on severity of primary dysmenorrhea
}

\author{
This article was published in the following Dove Press journal: \\ Patient Preference and Adherence \\ 10 February 2012 \\ Number of times this article has been viewed
}

\author{
Shahla Gharloghi' \\ Shahnaz Torkzahrani² \\ Ali Reza Akbarzadeh ${ }^{3}$ \\ Reza Heshmat ${ }^{4}$ \\ 'Department of Midwifery, School \\ of Nursing and Midwifery, \\ Kermanshah University of Medical \\ Sciences, Kermanshah, ${ }^{2}$ Department \\ of Midwifery, School of Nursing \\ and Midwifery, Shahid Beheshti \\ University of Medical Sciences, \\ ${ }^{3}$ Department of Biostatistics, School \\ of Health, Shahid Beheshti University \\ of Medical Sciences, Evin, Tehran, \\ IR Iran; ${ }^{4}$ International University for \\ Acupuncture, Lyon, France
}

Background: Dysmenorrhea constitutes one of the most frequent disorders in women of a fertile age. The objective of this research was to determine the effects of acupressure at Sanyinjiao (SP6) point and DiJi (SP8) point on pain severity of primary dysmenorrhea and the associated systemic symptoms.

Materials and methods: In this crossover clinical trial, 50 females aged 18-30 years old who met the study criteria and were under the care of Sarpolezahab Health Center were selected. Subjects were randomly assigned to one of two groups and evaluated during three menstrual cycles. We evaluated pain severity using the McGill pain scale and associated systemic symptoms using a verbal multidimensional scoring system. Data acquired from 42 cases were analyzed using SPSS software, with a $P$ value of $<0.05$ considered significant.

Results: The findings of our study indicate that the severity of dysmenorrhea pain diminishes significantly for up to 2 hours following treatment with acupressure at the SP6 and SP8 points $(P<0.001)$. Furthermore, the severity of associated systemic symptoms reduced significantly after acupressure at the SP6 and SP8 points, except for nausea and vomiting. Comparison of the severity of systemic symptoms with acupressure at the SP6 and SP8 points revealed no significant difference except for severity of fatigue, which was reduced significantly further with SP6 point compared to SP8 point $(P=0.004)$.

Conclusion: Acupressure at the SP6 and SP8 points can reduce pain severity of dysmenorrhea for up to 2 hours after application, and these points may be used to alleviate the severity of systemic symptoms accompanying dysmenorrhea.

Keywords: dysmenorrhea, systemic symptoms, acupressure, SP8 point, SP6 point

\section{Introduction}

Dysmenorrhea constitutes one of the most frequent fertility disorders, presenting as either primary or secondary. The former is defined as painful menstruation without pelvic pathology, which usually develops 1 or 2 years after menarche. It afflicts young women but may persist in females as old as 40 years of age. ${ }^{1}$ Dysmenorrhea affects almost half of all women, with symptoms becoming so severe in $10 \%$ of them as to disrupt their routine activities. ${ }^{2}$ Although primary dysmenorrhea is not fatal and causes no organ failure, it may affect the patient's life and result in disability and inefficiency. ${ }^{3}$ Complications of dysmenorrhea include disruption of routine activities; absence from school or workplace; medication use; physical and mental disorders; and social, economic, or familial problems. ${ }^{4}$ In the USA, an annual loss of 600 million hours and US\$2 billion is attributable to dysmenorrhea. ${ }^{5}$ Moreover, dysmenorrhea influences young girls' opinion of femininity and even the method of childbirth chosen. ${ }^{6}$
Correspondence: Shahnaz Torkzahrani Department of Midwifery, Shahid Beheshti University of Medical Sciences, Evin, Tehran, IR Iran

$\mathrm{Tel}+\mathrm{I} 982188202512$

Fax + I 982188202523

Email zahranishahnaz@yahoo.com 
Generally, treatment options for dysmenorrhea include both medical and non-medical options. Nonsteroidal anti-inflammatory drugs and oral contraceptive pills are the most common pharmacologic treatments for primary dysmenorrhea ${ }^{1,7}$ however, despite the fact that these medications may provide immediate pain relief, their use entails possible adverse reactions, drug dependence, and unnecessary medical expenses. ${ }^{8}$ Nonmedical options include bed rest, exercise, heating, and herbal medicines. ${ }^{9}$ One therapeutic option for dysmenorrhea is using traditional Chinese medicine, such as acupuncture, which has been used to treat acute and chronic pains including dysmenorrheal. ${ }^{5}$ Some researchers have suggested that manipulating the small myelinated peripheral nerve fibers in muscles with needles dispatches signals to spinal cord, midbrain, and the pituitary axis, resulting in release of substances such as enkephalin, dynorphin, serotonin, norepinephrine, and beta-endorphines, among others, into the bloodstream and cerebrospinal fluid, thus alleviating pain. ${ }^{10}$

Acupressure is an alternative form of acupuncture in which physical pressure is applied to the same energy points and channels that are used in acupuncture. ${ }^{5}$ Traditional Chinese philosophy holds that certain channels in the human body (called Qi) regulate the flow of energy and it is the unbalanced flow of Qi that results in disease. Acupuncture is believed to open the channels and balance energy, thus restoring health to the body. ${ }^{10}$

The Sanyinjiao (SP6) point is one of many points used in acupressure, particularly for gynecologic, genitourinary, and digestive disorders, as well as dystocia and alleviating labor pain. ${ }^{10,11}$ It is located on the medial aspect of the calf, three cuns (the breadth of patient's thumb) above the medial malleolus posterior to the border of tibia, on the spleen meridian. ${ }^{10}$

The DiJi (SP8) point is another point used in acupressure as emergency point of pain relief, particularly for abdominal pain, bloating, diarrhea, dysuria, dysmenorrhea, and irregular menses. ${ }^{12}$ It is among the best points for treating any type of dysmenorrhea and is probably the optimal point for dysmenorrhea caused by stasis. It is located on the line joining the malleolus to the Sanyinjiao (SP6) point, on the medial-lateral aspect of the calf, below the inferior margin of tibia and gastrocnemius, three cuns below the SP9 point on the spleen meridian. ${ }^{13}$

Previous studies have indicated that acupressure at the SP6 point may be considered as a noninvasive method for alleviating primary dysmenorrhea, with its effects persisting as long as 2 hours after treatment. ${ }^{8,14}$ Considering that, as far as the authors are aware, no study has yet been conducted to assess acupressure at SP8 point for alleviating dysmenorrhea, despite the fact that acupuncture textbooks present it as effective for reducing menstrual pain, ${ }^{12,13}$ and that insufficient studies have been conducted to compare the effects of acupressure at different points on primary dysmenorrhea, we undertook the present study to compare the possible differences in effects of acupressure at the SP6 and SP8 points on primary dysmenorrhea.

\section{Methods \\ Participants}

This study was a crossover clinical trial. Health care personnel invited females aged 18-30 years old who were under the care of the Sarpolezahab Health Center to attend a meeting at the center. Once the objectives of the study were explained to them, 150 females who suffered from dysmenorrhea and wished to participate in the study were evaluated. According to our inclusion criteria, participants: were single females with a minimum education level of high school diploma, who suffered from primary dysmenorrhea with a severity of moderate to severe (as determined by McGill scale); had regular menses with periods of 21-35 days; had not been diagnosed for documented chronic disease; did not mention symptoms such as itching, burning or abnormal discharge; did not have a history of pelvic inflammatory disease, myoma and tumors, pelvic infection, or ovarian cyst; and had not used, in the 6 months prior to the study, an oral contraception pill, an intrauterine device, or any particular medicine. In addition, participants did not smoke and did not mention having experienced a stressful event, such as parental separation or death of a first-degree relative, in the last 6 months.

We used power analysis to determine the sample size. Extrapolating for a medium size effect of 0.50 , a minimum sample of 42 subjects was needed to achieve a power of 0.80 with an alpha of 0.05 . However, taking into account the possible $20 \%$ sample loss, a total of 50 samples were selected. Therefore, 50 participants who met the inclusion criteria and provided informed consent were randomized using a computer-based randomization program procedure to randomly assign 25 individuals to either group A or B. Ultimately, from each group, four patients were excluded due to Ramadan fasting, loss to follow-up, marriage, or development of severe vaginal discharge. In the final sample, there were 21 participants in each group who were evaluated for three menstrual cycles (Figure 1).

\section{Procedures}

After allocation of participants to each group (A or B), subjects were taught acupressure techniques by a researcher. 


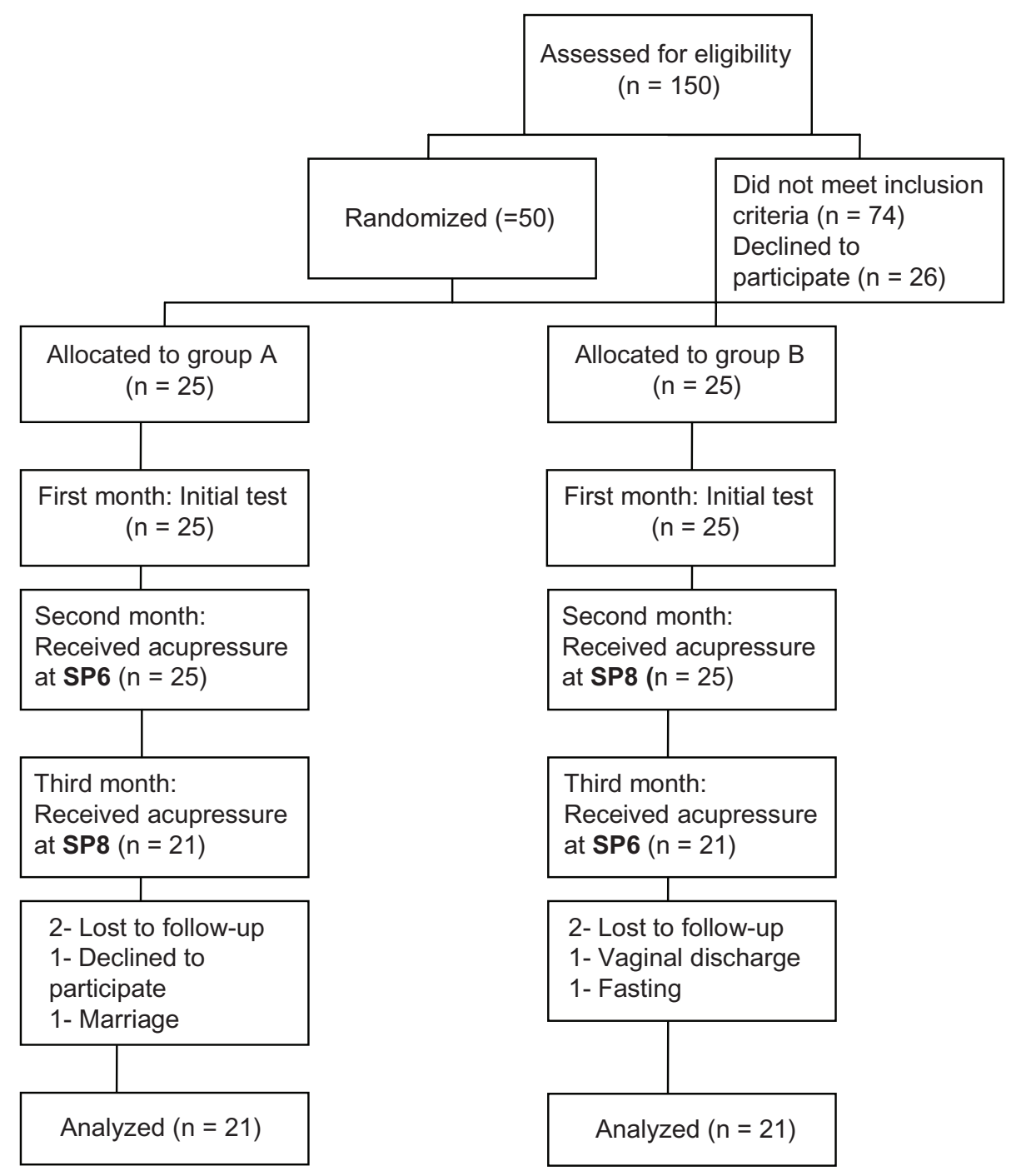

Figure I Flow diagram of participant progress.

Abbreviations: SP6, Spleen 6 (or Sanyinjiao); SP8, Spleen 8 (or Diji).

Participants were instructed on the correct methods of locating either the SP6 or SP8 point and applying pressure; thus, as soon as pain started, each participant was instructed to press the specified point with her thumb in a clockwise circular fashion for 5 minutes, then press the point on the other foot for 5 minutes, this time in a counterclockwise fashion, and then repeat the whole procedure to yield a total period of 20 minutes; two periods of 5 minutes spent on each foot. After applying acupressure, participants were allowed to use any additional conventional treatments as needed. The procedures were applied once per day during first 3 days of the menstruation period. The instruction took place at the site of the health care center and continued until the researcher was convinced of the learner's correct location and manipulation of the points. Accuracy of SP6- and SP8point identification was validated with researcher who was taught by specialist in acupressure for more than six sessions over a period of 1 month.

Participants completed standardized questionnaires that included items on sociodemographic characteristics and information regarding severity of dysmenorrhea, systemic symptoms, and other issues related to menses at baseline and through the first 3 days of the first menstrual cycle (average duration of menstrual pain) and for two consecutive cycles. The main outcome parameter was average pain intensity, which was measured on a numeric rating McGill scale $^{15}$ ranging from 0 to 10 . The proper method of using the McGill scale was explained to the participants by the researcher. On this scale, a score of 1-3 indicates mild pain, 4-7 moderate pain, and 8-10 severe pain. The participants completed the part pertaining to the McGill pain scale before therapy, immediately after therapy and then 
30 minutes, 1 hour, and 2 hours after applying acupressure. They also completed the part pertaining to verbal multidimensional system for evaluation of systemic symptoms before therapy and 2 hours after applying acupressure. The verbal multidimensional scoring system for evaluation of systemic symptoms is graded from 0 to 3: 0 (no symptoms); 1 (symptoms exist only mildly and do not interfere with routine activities); 2 (symptoms exist moderately and interfere with routine activities but are not debilitating); and 3 (symptoms exist severely and are completely debilitating).

Through 3 months of follow-up, on first menstrual cycle as an initial assessment, information about severity of dysmenorrheal and systemic symptoms was obtained from both groups. On the second menstrual cycle, group A applied acupressure at SP6 and group B at SP8 point. On the third menstrual cycle, group A applied acupressure at SP8 and group B at SP6 point. Data was obtained by questionnaire (in Farsi [Persian]) and their validity and reliability were determined by content validity and test re-test, respectively. The participants were asked to complete the questionnaires once per day. All questionnaires were gathered by the researcher at end of each month.

\section{Data analysis}

Data were analyzed by Wilcoxon as a nonparametric test to compare two paired groups. The severity of pain and symptoms through variety of time and cycle were also compared by Friedman test. The accepted level of significance for all other analyses was $P<0.05$. Data analyses were carried out using SPSS (v 16; IBM Corp, Endicott, NY).

\section{Results}

The findings in this study indicate that $73.3 \%$ of participants were aged between 18 and 22 years, their mean age was 21.14 $( \pm 2.88)$ years, $71.4 \%$ had high school diploma, and $28.6 \%$ had university education. Of the participants, $2.4 \%$ had mild, $59.5 \%$ moderate, and $38.1 \%$ severe menstruation periods. There was no significant difference between the two groups regarding menstruation period severity, age, and education.
As Table 1 demonstrates, mean (standard deviation [SD]) pain severity in the group using the SP6 point was 6.56 $( \pm 1.05)$ prior to acupressure, which diminished to $5.69( \pm 1.14)$ immediately after acupressure, $5.20( \pm 1.25) 30$ minutes after acupressure, $4.20( \pm 1.41) 1$ hour after acupressure, and 2.89 $( \pm 1.36) 2$ hours after acupressure. In addition, mean $(S D)$ pain severity in the group using the SP8 point was $6.84( \pm 1.09)$ prior to acupressure, which diminished to $4.47( \pm 0.99)$ immediately after acupressure, $4.08( \pm 1.01) 30$ minutes after acupressure, $3.17( \pm 0.96) 1$ hour after acupressure, and $2.03( \pm 0.94)$ 2 hours later. The reductions in pain severity were significant for all intervals and at both SP6 and SP8 points $(P<0.001)$. Comparison of the acupressure applied at SP6 and SP8 points revealed that, for all intervals, acupressure at the SP8 point reduced pain severity significantly more than the SP6 point $(P<0.001)$.

Mean severity of systemic symptoms of participants before and after acupressure at the SP6 and SP8 points is shown in Table 2 . The severity of seven systemic symptoms accompanying dysmenorrhea diminished significantly after application of acupressure at the SP6 point. Similarly, the severity of all these symptoms, except nausea, diminished significantly with acupressure at the SP8 point. Comparing the severity of systemic symptoms between acupressure at the SP6 and SP8 points revealed no significant difference, except for fatigue, which was alleviated significantly more with SP6 than SP8 $(P=0.004)$.

\section{Discussion}

In the present study, we divided 50 patients randomly into two groups. During the first cycle, we determined the severity of dysmenorrhea for each group prior to intervention. During the next two cycles, each group experienced, alternatively, acupressure once at SP6 point and once at SP8 point. Since in this type of clinical trial (crossover trial) each group is once the intervention group and once the control group, the confounding factors are almost completely under control. Therefore, the crossover clinical trial is a powerful clinical study. Our

Table I Pain severity experienced by subjects before, immediately, 30 minutes, I hour, and 2 hours after acupressure at the Sanyinjiao (SP6) and Diji (SP8) points

\begin{tabular}{llllll}
\hline Cycle & \multicolumn{2}{l}{ Mean and standard deviation of pain severity } & & \\
\cline { 2 - 6 } & Before acupressure & $\begin{array}{l}\text { Immediately after } \\
\text { acupressure }\end{array}$ & $\begin{array}{l}\mathbf{3 0} \text { minutes after } \\
\text { acupressure }\end{array}$ & $\begin{array}{l}\text { I hour after } \\
\text { acupressure }\end{array}$ & $\begin{array}{l}\mathbf{2} \text { hours after } \\
\text { acupressure }\end{array}$ \\
\hline SP6 & $6.56 \pm 1.05$ & $5.69 \pm 1.14$ & $5.20 \pm 1.25$ & $4.20 \pm 1.4 \mathrm{I}$ & $2.89 \pm 1.36$ \\
SP8 & $6.84 \pm 1.09$ & $4.47 \pm 0.99$ & $4.08 \pm 1.01$ & $3.17 \pm 0.96$ & $2.03 \pm 0.94$ \\
Comparing SP6 and SP8 & NS & $P<0.001$ & $P<0.001$ & $P<0.001$ & $P<0.001$ \\
\hline
\end{tabular}

Abbreviation: NS, not significant. 
Table 2 Mean severity of systemic symptoms of study units before and after acupressure at Sanyinjiao (SP6) and Diji (SP8) points

\begin{tabular}{|c|c|c|c|c|c|}
\hline \multirow[t]{2}{*}{ Systemic symptoms } & \multicolumn{3}{|c|}{ Mean and SD severity of systemic symptoms } & \multicolumn{2}{|c|}{ Comparison of cycles ( $P$ values) } \\
\hline & Before acupressure & SP6 & SP8 & $\begin{array}{l}\text { Before acupressure } \\
\text { and SP6 }\end{array}$ & $\begin{array}{l}\text { Before acupressure } \\
\text { and SP8 }\end{array}$ \\
\hline Exhaustion & $0.87 \pm 0.58$ & $0.25 \pm 0.30$ & $0.13 \pm 0.20$ & $<0.00$ I & $<0.001$ \\
\hline Vomiting & $0.11 \pm 0.28$ & $0 \pm 0$ & $0.02 \pm 0.11$ & 0.010 & NS \\
\hline Paralysis & $0.96 \pm 0.75$ & $0.10 \pm 0.20$ & $0.05 \pm 0.14$ & $<0.001$ & $<0.001$ \\
\hline Diarrhea & $0.08 \pm 0.32$ & $0 \pm 0$ & $0 \pm 0$ & 0.039 & 0.039 \\
\hline Headache & $0.25 \pm 0.36$ & $0.007 \pm 0.05$ & $0.03 \pm 0.16$ & $<0.001$ & $<0.001$ \\
\hline Neural & $0.61 \pm 0.65$ & $0.007 \pm 0.17$ & $0.03 \pm 0.13$ & $<0.001$ & 0.001 \\
\hline Faint & $0.21 \pm 0.52$ & $0.007 \pm 0.05$ & $0.02 \pm 0.11$ & 0.005 & 0.016 \\
\hline
\end{tabular}

Abbreviation: SD, standard deviation.

findings indicate that the severity of dysmenorrhea diminished significantly immediately, 30 minutes, 1 hour, and 2 hours after application of acupressure at the SP6 and SP8 points. Previous studies indicate that acupressure at the SP6 point alleviates dysmenorrheal pain. ${ }^{14,16-19}$ In a study similar to the present study, Jun et al demonstrated the effects of SP6 acupressure on dysmenorrheal pain in women by series of measurement time points (ie, immediately, 1, 2, and 3 hours post-treatment) and by blinding the participants to the treatment. ${ }^{8}$ In contrast, our study was not blinded. Moreover, in this study the severity of dysmenorrhea was determined within 2 hours after applying acupressure. In Jun et al's study, there was a significant difference in severity of dysmenorrhea between the two groups of intervention (acupressure at the SP6 point) and control (placing the thumb on the SP6 point slowly with no pressure) immediately after and for up to 2 hours after applying acupressure, but no difference was observed between the two groups at 3 hours after applying acupressure. ${ }^{8}$

Wong et al reported that acupressure has a long-term and accumulative effect in relieving primary dysmenorrhea. ${ }^{17}$ They showed that SP6 acupressure has an immediate painrelieving effect and a significant pain reduction in the third month. However, our study failed to show that SP6 acupressure had effectiveness over a long time period because the two groups received acupressure at the SP6 point for one cycle and the SP8 point for one cycle. Also, the effects reported in our study were similar to findings in previous literature where there was a statistically significant reduction in the intensity of pain and anxiety of dysmenorrhea when acupressure at the SP6 point was applied. ${ }^{14}$ However, in our study, anxiety of dysmenorrhea was not determined. As for acupressure at the SP8 point, we were unable to find a similar study that has evaluated its effects on dysmenorrhea.

The findings of this study indicate the possible physiological mechanism of acupressure, such as decreasing pain through activation of an endogenous opioid system and release of specific neuropeptides ${ }^{20}$ and restoring health to body through balance of energy. ${ }^{10,21}$

Another result of this study is the more efficacious pain relief experienced with the SP8 point compared with the SP6 for different intervals. The SP8 point is considered the emergency point for pain relief; it constitutes one of the best points for treating dysmenorrhea of any type and is probably the optimal point for treating dysmenorrhea caused by stasis. ${ }^{12,13}$ This study also corroborates the better efficacy of this point for relieving menstrual pain; however, further studies are required to allow for a more confident conclusion.

Furthermore, our findings indicate that systemic symptoms accompanying dysmenorrhea diminish significantly with acupressure at both points - except for nausea at the SP8 point. The SP6 and SP8 points did not differ significantly in their efficiency for reducing these systemic symptoms, except in the case of fatigue, which diminished further with the SP8 point compared with the SP6. Previous studies also report the ability of acupressure to alleviate systemic symptoms. ${ }^{5,14,22}$ Smith et al conducted a study in 2011 and concluded that acupressure and acupuncture are more efficient in relieving systemic symptoms compared with medical therapy and traditional Chinese herbal medicines. $^{23}$

\section{Conclusion}

Considering the findings of the present study, it appears that acupressure at the SP6 and SP8 points alleviates menstrual pain. The resting period was 2 hours after application and was more pronounced with the SP8 point compared with the SP6. Therefore, the SP8 point can be considered an emergency point for relieving primary dysmenorrhea. Moreover, acupressure reduces the severity of systemic symptoms of dysmenorrhea. Given these effects of acupressure, it may be used as a convenient self-care method for different patients, free of cost and complications. To the authors' knowledge, this is the first controlled study to provide evidence that acupressure at 
the SP8 point may be effective for the treatment of primary dysmenorrhea. Therefore, we recommend the conduct of further studies using acupressure at the SP8 point for different periods and cycles and on larger populations.

\section{Study limitations}

Due to the type of study this was and the self-administration of acupressure by the participants, neither providers nor participants were blinded to treatment. Therefore, unblinding bias cannot be ruled out. Some common limitations of the crossover trial design such as carry-over (previous treatment) and order effects may affect studies of this kind. Carryover effect is unlikely to apply in this study because of the 1-month interval between treatment of the two groups, but it is possible that the order in which the SP6 or SP8 points were used as treatment affected the outcomes in this study. The attrition rate was not excessive (16.7\%), but attrition bias could accrue; however, the characteristics of those participants who dropped out were similar to those who completed the follow-up.

\section{Acknowledgments}

This study was an MSc dissertation. Thereby, we express our gratitude to authorities of Shahid Beheshti University of Medical Sciences. We also thank the participants in the trial who helped us in conducting this research.

\section{Disclosure}

The authors declare no conflicts of interest in this work.

\section{References}

1. Berek JS, Novak E. Dysmenorrhera. Novak's Gynecology. 14th ed. Philadelphia, PA: Lippincott Williams and Wilkins; 2007:391-393.

2. Daniels SE, Torri S, Desjardins P. Valdecoxib for treatment of primary dysmenorrhea. J Gen Intern Med. 2005;20(1):62-67.

3. Whittle GC, Slade P, Ronalds CM. Social support in women reporting dysmenorrhea. J Psychosom Res. 1987;31(1):79-84.

4. Pour Eslami M, Amin A, Sarmast H. Assessment of knowledge, attitudes and behavior of adoloescent girls in suburban districts of Tehran regarding dysmenorrhea and menstrual hygiene. Journal of Shahid Sadoughi University of Medical Sciences and Health Services. 2002;9(4):23-29.
5. Taylor D, Miaskowski C, Kohn J. A randomized clinical trial of the effectiveness of an acupressure device (relief brief) for managing symptoms of dysmenorrhea. J Altern Complement Med. 2002;8(3):357-370.

6. Teperi J, Rimapelä M. Menstrual pain, health and behavior in girls. Soc Sci Med. 1989;29(2):163-169.

7. Zhang WY, Li Wan Po A. Efficacy of minor analgesics in primary dysmenorrhea: a systematic review. Br JObstet Gynaecol. 1998;105(7): 780-789.

8. Jun EM, Chang S, Kang DH, Kim S. Effects of acupressure on dysmenorrhea and skin temperature changes in college students: a nonrandomized controlled trial. Int J Nurs Stud. 2007;44(6):973-981.

9. Akin MD, Weingand KW, Hengehold DA, Goodale MB, Hinkle RT, Smith RP. Continuous low-level topical heat in the treatment of dysmenorrhea. Obstet Gynecol. 2001;97(3):343-349.

10. Heshmat R, Rostami U. Acupressure and Health Moxibustion in Family Fertility Health and Infertility Treatment. 1st ed. Tehran: Teimorzadeh; 2009. Persian.

11. Gentz BA. Alternative therapies for the management of pain in labor and delivery. Clin Obstet Gynecol. 2001;44(4):704-732.

12. Cheng K. Chinese Acupuncture and Moxibustion. 1st ed. Foreign Languages Press; 1999:150.

13. Sionneau P. Acupuncture. Les Points Essentiels [Acupuncture. The essential points]. Trédaniel G, editor. Paris: Guy Trédaniel; 2000. French.

14. Chen HM, Chen CH. Effects of acupressure at the Sanyingiao point on dysmenorrhea. $J A d v$ Nurs. 2004;48(4):380-387.

15. Melzack R. The McGill Pain Questionnaire: major properties and scoring methods. Pain. 1975;1:277-299.

16. Chang SB, Jun EM. Effects of SP-6 acupressure on dysmenorrhea, cortisol, epinephrine and norepinephrine in the college students. Taehan Kanho Hakhoe Chi. 2003;33(7):1038-1046. Korean.

17. Wong CL, Lai KY, Tse HM. Effects of SP6 acupressure on pain and menstrual distress in young women with dysmenorrhea. Complement Ther Clin Pract. 2010;16(2):64-69.

18. Mirbagher-Ajorpaz N, Adib-Hajbaghery M, Mosaebi F. The effects of acupressure on primary dysmenorrhea: a randomized controlled trial. Complement Ther Clin Pract. 2011;17(1):33-36.

19. Kashefi F, Ziyadlou S, Khajehei M, Ashraf AR, Fadaee AR, Jafari P. Effects of acupressure at the Sanyingiao point on primary dysmenorrhea: a randomized controlled trial. Complement Ther Clin Pract. 2010;16(4):198-202.

20. Ernst E, Fialka V. The clinical effectiveness of massage therapy a critical review. Forsch Komplementarmed. 1994;1:226-232.

21. Navidi AA, Khedmat H, Helli Saz MT, Naseri MH, Ghasemi Salmanie KH. Basic Principles and Clinical Acupuncture. Tehran: Tabib Publications; 2006.

22. Wang MC, Hsu MC, Chien LW, Kao CH, Liu CF. Effects of auricular acupressure on menstrual symptoms and nitric oxide for women with primary dysmenorrhea. J Altern Complement Med. 2009; 15(3):235-242.

23. Smith CA, Zhu X, He L, Song J. Acupuncture for primary dysmenorrhoea. Cochrane Database Syst Rev. 2011;1:CD007854.
Patient Preference and Adherence

\section{Publish your work in this journal}

Patient Preference and Adherence is an international, peer-reviewed, open access journal focusing on the growing importance of patient preference and adherence throughout the therapeutic continuum. Patient satisfaction, acceptability, quality of life, compliance, persistence and their role in developing new therapeutic modalities and compounds to

\section{Dovepress}

optimize clinical outcomes for existing disease states are major areas of interest. This journal has been accepted for indexing on PubMed Central. The manuscript management system is completely online and includes a very quick and fair peer-review system. Visit http://www.dovepress.com/ testimonials.php to read real quotes from published authors. 